BACTERIAL DNA

\section{Coli Coil}

from our Molecular Biology Correspondent

AN important development, which should provide a new direction for studies on control of transcription, has been described by Stonington and Pettijohn (Proc. US Nat. Acad. Sci., 68, 6; 1971). What they have done is to isolate as defined particles the nuclear bodies of Escherichia coli. The secret lies in the use of fresh exponential phase cells, gentle lysis with lysozyme and rapid isolation of the particles by a brief centrifugation through a sucrose gradient. The product after extraction from the cytoplasm is stable, sediments at $3200 \mathrm{~S}$ and has the low viscosity expected for a compact globule. It comprises 80 per cent DNA, the remainder being RNA and protein. The RNA is known from earlier work to be made up in the main of growing messenger and ribosomal chains. The protein is almost entirely RNA polymerase, and comparison of the gel electrophoresis pattern with that of the standard polymerase preparation reveals the presence of the $\alpha$, $\beta$ and $\beta^{\prime}$ chains, but not of $\sigma$-factor, in accordance with the current view that this is released after initiation.

Treatment of the complex with ribonuclease causes it to erupt into linear DNA with a huge increase in viscosity. The same effect is elicited by sodium dodecyl sulphate, but not by proteolytic enzymes. It thus seems that the RNA plays a part in stabilizing the particle and the same may or may not be true of the protein. The nuclear particles are reportedly good templates for RNA polymerase, and it is unavoidable to inquire what attributes they might have in common with chromatin, the properties of which are allegedly controlled in part by endogenous RNA. The observations of Stonington and Pettijohn also add point to the attempts of Lerman and his colleagues-described recently at a meeting of the British Biophysical Society (see Nature, 229, 12; 1971) and in an abstract (Proc. US Nat. Acad. Sci., 66, 242; 1970) - to characterize a compact form of DNA generated under special conditions selected to simulate them in the cell.

A compact, supercoiled DNA is also found in plasmids of $E$. coli, which are extra-chromosomal bodies, containing a small proportion of protein. I recently drew attention to the work of Clewell and Helinski on one of these (colicinogenic factor $E_{1}$ ), in which it was shown that the supercoil winding was released by introduction of a single strand break apparently by a latent nuclease associated with the DNA. These observations have now been extended in work from the same laboratory (Blair et al., ibid., 68, 210 ; 1971) to another colicinogenic factor, $E_{2}$. As with $E_{1}$, treatment with

pronase, or a denaturing detergent, inhibited the relaxation effect. By contrast with $E_{1}$, heat treatment of $E_{2}$ substantially prevented relaxation, while leading to the loss of the protein from the supercoiled fraction. This seems to establish beyond reasonable doubt that the DNA in the plasmid is associated with a nicking enzyme that can be inactivated by proteolysis or denaturation.

In order to determine whether the break occurs in the light ("Watson") or heavy ("Crick") strand, Blair et al. have made use of a procedure introduced by Hradeczna and Szybalski involving isopycnic centrifugation in the presence of poly (U,G), which has high affinity for the heavy strand. The circular and linear strands are first separated by sucrosegradient sedimentation at alkaline $p \mathrm{H}$. In this way it was established that Watson is intact and Crick ruptured. A single-strand break must be supposed to precede replication, and it is to be noted

\section{New Assays for Old}

ONE of the advantages of conventional radioimmunoassay is its high specificity -the antibody produced against the hormone concerned frequently shows little or no cross-reaction with quite closely related compounds. This feature is particularly important in biological assay because in vivo and in vitro degradation products are frequently present in extracts. Equally, however, if there are closely related compounds of interest in addition to the one under study, this antibody specificity necessitates producing separate specific antisera for immunoassay-or at least this is the conventional approach.

Giese, Nielsen and Jørgensen now show in next Wednesday's Nature New Bio$\log y$ that this approach may not be essential. They have taken advantage of the heterogeneity of antibodies for assay of materials which are apparently noncross-reacting. Their investigation concerned antibodies against angiotensins I and II which do not usually cross-react when tested by conventional assay. Thus, displacement of labelled angiotensin II from its specific antibody by unlabelled angiotensin $I$ is usually less than 1 per cent. Giese et al., however, now show that if the same anti-angiotensin II antibody is tested using labelled angiotensin I, not only do quite a high proportion of sera bind the label (albeit at a much lower dilution) but also that this binding is relatively specific; that is the labelled angiotensin I is displaced from antibody much more readily by unlabelled angiotensin I than by unlabelled angiotensin II.

The practical consequence of this in angiotensin immunoassay may be that radioimmunoassay of angiotensin $I$ can be performed using the more readily that Gilbert and Dresser have given evidence that the strand broken in induction of $\lambda$-prophage is also the Crick strand.

For anybody with a serious interest in supercoiling, another surpassingly accomplished article by Bauer and Vinograd (J. Mol. Biol., 54, 281; 1970) is mandatory reading. This concerns the relation between supercoiling, as controlled by dye intercalation, and buoyant density. The insertion of ethidium bromide into the DNA double helix has two effects. One is that the helix behaves as a stiffer and a more slender rod in solution. The second is that the intercalation causes a partial uncoiling of the helix at the point of insertion, and in closed circular DNAs this causes a reduction in the degree of supercoiling which in turn changes the buoyant density of DNA. The diagnostic application of the phenomenon for determining whether drugs, antibiotics and the like bind by intercalation or otherwise is developed by Waring (ibid., 247). available anti-angiotensin II sera. It will be of great interest to see whether this system can be extended to other antibodies, for if the sensitivity and specificity of assay can be made high enough this could be a very important contribution to the assay of closely related compounds such as steroids or peptide hormones of different species, particularly where antisera are difficult to raise.

$\mathrm{Ng}$ and Vane showed in 1967 (Nature, 216, 762) that it is the lung and not the blood which is the important site of angiotensin I conversion in vivo to the biologically active component of the renin angiotensin system, angiotensin II. Bakhle and Reynard have now taken steps towards characterization of a converting enzyme in the lung by isolating from lung homogenates a material which is relatively free from enzymes which destroy angiotensin, collectively referred to as "angiotensinases". In next Wednesday's Nature New Biology, they present some of their preliminary data on its characteristics as tested in their in vitro assay system, with particular reference to its metal dependence, $p \mathrm{H}$ optimum and relationship to plasma converting enzyme. Several features of some interest emerge at this stage, in particular the observation that converting enzyme activity is apparently membrane bound. Moreover, it seems to have a relatively slow rate of angiotensin I converting activity when compared with the highly active in vivo system. But it would be premature to draw too firm conclusions at this stage and further understanding must await better purification. Precise specificity studies are also needed to ensure that this material is the same as the mechanism responsible for in vivo angiotensin I conversion. 